\title{
Cuerpo, acontecimiento y política en Gilles Deleuze
}

\author{
Body, event and politics in Gilles Deleuze
}

\author{
FRANCISCO JAVIER ALCALÁ RODRÍGUEZ*
}

\begin{abstract}
Resumen: El presente trabajo pretende analizar la reducción del cuerpo a los caracteres de Lo Mismo en que está escrito el mundo verdadero de la metafísica, tal y como es llevada a cabo por la Imagen Dogmática del pensamiento denunciada por Gilles Deleuze. Desde esta perspectiva, se intentará desmontar la supuesta inocencia axiológica de la Imagen Dogmática del pensamiento, bosquejando en primer lugar las indeseables consecuencias políticas que la misma lleva aparejadas y tratando en segundo lugar de dilucidar las condiciones de posibilidad del cambio sociopolítico en la filosofía de Gilles Deleuze y Félix Guattari.

Palabras clave: acontecimiento, cuerpo, política, epistemología, axiología, Imagen Dogmática.
\end{abstract}

\begin{abstract}
This work pretends to analyse the reduction of the body to the characters of The Same in which is written the true world of metaphysics, in the way in which it has been done by the Dogmatic Image of Thought denounced by Gilles Deleuze. From this perspective, we will try to dismantle the supposed axiological innocence of the Dogmatic Image of Thought, elucidating in first place its undesirable political consequences and trying in second place to analyse the conditions of possibility of socio-political change in Gilles Deleuze and Félix Guattari's philosophy. Keywords: event, body, politics, epistemology, axiology, Dogmatic Image.
\end{abstract}

\section{Introducción. El cuerpo y la imagen dogmática del pensamiento}

Quizá toda aproximación certera al inabarcable sistema filosófico debido a Gilles Deleuze haya de considerar detenidamente la apropiación del proyecto nietzscheano de

Fecha de recepción: 11/06/2016. Fecha de aceptación: 17/07/2016.

* Francisco Javier Alcalá Rodríguez (Antequera, 1985) es Licenciado en Filosofía por la Universidad de Málaga y Máster en Filosofía Contemporánea por la Universidad de Granada. Actualmente, es investigador predoctoral del programa de Formación de Profesorado Universitario del Ministerio de Educación (código de ayuda: FPU13-05570) en el departamento de Filosofía II de la Universidad de Granada, bajo la dirección del profesor José Antonio Pérez Tapias. Recientemente, ha publicado los artículos "Epistemología y axiología en Gilles Deleuze. Del pensar sin presupuestos a la crítica genealógica de cuño nietzscheano", en Claridades Revista de Filosofía 5 (2013), pp. 79-100; y "De luces y sombras: la dualidad «naturaleza-civilización» en la pintura de Caspar Friedrich como antecedente de «El corazón de las tinieblas», de Joseph Conrad”, en González Fisac, Jesús (ed.), Barbarie y civilización: XVI Encuentro de la Ilustración al Romanticismo: Cádiz, América y Europa ante la modernidad. 1750-1850, Servicio de Publicaciones de la Universidad de Cádiz: Cádiz, 2015, pp. 33-47. Sus líneas de investigación se inscriben en el marco de la filosofía contemporánea y, especialmente, en el pensamiento de Gilles Deleuze. 
"invertir el platonismo" que llevó a cabo el pensador francés en el primer apéndice de Lógica del Sentido (Deleuze, 2003, "Platón y el simulacro"); ${ }^{1}$ proyecto a que se alude frecuentemente, en éstos u otros términos, a lo largo de toda su obra posterior. Invertir el platonismo sería, entonces, el afán que recorre de principio a fin la filosofía deleuzeana, otorgando cierta unidad a su abigarrada producción intelectual, cuya complejidad rizomática sugiere un carácter no menos laberíntico que evolutivo.

Tal proyecto consiste, ante todo, en denunciar el gesto tácito con que Platón se aseguró de que la sombra de su filosofía fuese alargada y pesara como una losa sobre la historia del pensamiento posterior: la consabida distinción entre lo inteligible (Idea) y lo sensible (Imagen) que articulaba la filosofía platónica, relegando lo sensible al ámbito yermo de la apariencia, no era más que un pretexto para deslizar una segunda distinción más profunda y sutil; cuyo cometido no es otro que desplazar la primera hasta el ámbito de la apariencia sensible, blindando el platonismo contra toda crítica posterior no precavida. La distinción platónica entre lo inteligible y lo sensible es desplazada, pues, de forma tácita al ámbito de lo sensible, nuevo campo de batalla, con una intención inequívoca: asegurar el triunfo de las copias sobre los simulacros, es decir, el de aquellas imágenes que participan de la Idea (las cosas limitadas y medidas, que suponen paradas, establecimientos de presentes y asignaciones de sujetos; representadas en el lenguaje por los sustantivos y los adjetivos) sobre aquéllas que se hurtan a su acción (el devenir sin medida, que avanza en los dos sentidos a la vez, esquivando siempre el presente, disolviendo subjetividades y haciendo coincidir el futuro y el pasado; representado en el lenguaje por los verbos) (Cfr. Deleuze, 2005, 27-29). El auténtico sentido del método de la división no es, pues, dividir un género en especies, sino seleccionar al pretendiente verdadero entre los falsos pretendientes a partir de un Modelo o fundamento Ideal. De este modo, la metafísica se constituye como filosofía del ser renegando del devenir, con miras a asegurar de una vez por todas el ámbito de la representación que reconoce como propio.

De la caracterización precedente se sigue que la metafísica platónica reduce lo sensible a mundo verdadero, es decir, a una realidad pretendidamente exterior al pensamiento, dividida en objetos homogéneos e idénticos a sí mismos, cuyo garante es siempre un Modelo o fundamento Ideal (la Idea o esencia) susceptible de ser aprehendido por el pensar. Tales objetos son los reconocidos por el sujeto entendido como facultad de facultades, esto es, como una meta-facultad encargada de armonizar los contenidos de las restantes facultades corporales de acuerdo con los dictados del sentido común (el cuerpo es, por ende, tematizado como organismo). Lo que hay tras este planteamiento es una interiorización en filosofía del concepto de verdad que postula una relación natural entre la verdad y el pensamiento, de modo que bastaría con pensar naturalmente para pensar con verdad; constituyendo la llamada Imagen Dogmática del pensamiento, que opera en filosofía, al menos, desde Platón

1 En lugar del verbo francés inverser ("invertir"), Deleuze utiliza renverser, que recoge también los significados de "derrocar", en sentido político, y “atropellar": habla, pues, de «renverser le platonisme» (Cfr. Deleuze, 1969, 292-307). Podría decirse entonces que la traducción estándar al castellano de esta voz francesa no es del todo afortunada, pues en la filosofía deleuzeana se trata más bien de trastocar o subvertir el platonismo que de llevar a cabo una inversión del mismo; afán que, sin lugar a dudas, conduciría al nihilismo incompleto descrito por Nietzsche en el quinto estado del célebre pasaje "Historia de un error" (Cfr. Nietzsche, 1980, 52). Si bien, una vez introducida tan necesaria precisión, optamos por respetar la traducción habitual al castellano para no inducir a confusión al lector. 
(Cfr. Deleuze, 2006, "La imagen del pensamiento"; Zourabichvili, 2004, 14 y siguientes). Todos aquellos aspectos de lo sensible y lo corporal que se muestren rebeldes frente a la reducción descrita son relegados a una suerte de "exterioridad mala" del pensamiento, la cual es entendida como una fuerza externa venida a perturbarlo de forma accidental y provisoria, conduciéndolo al error. Tal exterioridad es estigmatizada como no-ser y denunciada como apariencia. Luego, entre las consecuencias indeseables que trae consigo la Imagen Dogmática del pensamiento se cuenta la relegación de los aspectos de lo corporal y lo sensible no reducibles a Lo Mismo al ámbito de la exterioridad mala o el error; única amenaza que gravitaría sobre todo aquél que piense a su abrigo.

Inserto en el marco teórico descrito, el presente trabajo parte de las siguientes hipótesis:

1. Lo sensible y el cuerpo son asumidos por la Imagen Dogmática sólo en apariencia; esto es, en la justa medida en que sean copias de un Modelo o fundamento Ideal que transcriba las intensidades diferenciales que los constituyen en los caracteres de Lo Mismo en que está escrito el mundo verdadero de la metafísica.

2. Aunque pueda parecer una mera opción epistemológica, la Imagen Dogmática del pensamiento tiene consecuencias en el plano axiológico.

Y tiene por objeto:

1. La dilucidación de las consecuencias que se siguen de la Imagen Dogmática del pensamiento a nivel socio-político; desde la doble perspectiva del saber, que fija las relaciones de poder, y la filosofía, forma particular del saber que atañe ante todo al pensamiento tal y como es de derecho.

2. Vislumbrar las condiciones de posibilidad del cambio socio-político (plano axiológico: relaciones de poder) en la ruptura con la Imagen Dogmática bajo todas sus formas, la cual supondría rechazar la reducción de lo corporal y lo sensible a Lo Mismo que ésta lleva aparejada en el plano epistemológico.

\section{Consecuencias socio-políticas de la imagen dogmática del pensamiento}

La clave de la inversión del platonismo a que Deleuze consagra su filosofía radica en la constitución de un pensamiento en que los cuerpos pasen a constituir el ser, en contraste con las filosofías de Platón, Aristóteles y sus herederos en la historia del pensamiento (Bréhier, 1928, 1-2). Los primeros en llevar a cabo tal proeza fueron, a su juicio, los audaces pensadores estoicos (Cfr. Deleuze, 2005, 33). En Lógica del sentido, Deleuze retomaba la división estoica entre las mezclas de cuerpos o las acciones y pasiones corporales, de un lado, y el sentido y los atributos o acontecimientos incorporales, del otro (Cfr. Deleuze, 2005, Series $2^{\mathrm{a}}, 4^{\mathrm{a}}, 5^{\mathrm{a}}$ ). Los segundos son efectos de superficie de las causas corporales, de manera que no puede decirse que sean en sentido estricto: más que existir, «insisten» en la superficie del ser. Ahora bien, es el descubrimiento de la casi-causa lo que determina la novedad del planteamiento estoico y su neta superioridad frente al epicúreo (Cfr. Deleuze, 2005, p. 126): es condición sine qua non de la distinción entre las causas corporales y sus efectos incorporales que los segundos devengan autónomos con respecto 
a las primeras; pues, de lo contrario, quedarían subsumidos en ellas, no gozando de mayor realidad que un fantasma o un espejismo. El producto deviene entonces productivo y se arroga una causalidad autónoma, cuyo ámbito de acción es la superficie del sentido: los atributos incorporales pueden actuar unos sobre otros, de acuerdo con esta nueva causalidad descubierta en la superficie de los cuerpos.

Conviene no pasar por alto, en este punto, que entre las mezclas de cuerpos y sus efectos incorporales hay una diferencia de naturaleza o heterogeneidad radical: son series heterogéneas o divergentes, la de las acciones y pasiones corporales y la de los atributos lógicos, cuya separación hay que observar de manera rigurosa para no caer en equívocos que restauren el indeseable idealismo que caracterizó a buena parte de la tradición filosófica, que abría un enunciado en la visibilidad y una visibilidad en el enunciado, invistiendo a los cuerpos y sus mezclas de sentido y viceversa (Deleuze, 2005, $6^{\text {a }}$ Serie). Fieles a este afán, los estoicos llevaron tal observancia hasta el exceso y la demencia: la espada que hiende y desgarra la carne en la batalla, provocando la muerte del guerrero, nada tiene que ver con el acontecimiento del morir; puro expresado de la proposición ("el guerrero muere", en este caso) que, no obstante, se atribuye a los cuerpos. Si bien, en Lógica del sentido Deleuze se distanciaba de los estoicos al caracterizar la instancia paradójica de la superficie como ámbito del sentido y la naturaleza igualmente paradójica del acontecimiento (puro expresado de la proposición que, no obstante, se atribuye a los cuerpos) como quintaesencia del lenguaje (Cfr. Deleuze, 2005 , series $2^{\mathrm{a}}, 3^{\mathrm{a}}, 31^{\mathrm{a}}$ ). Ambos vincularían, en sus ámbitos respectivos -sentido y lenguaje-, las series divergentes de acuerdo con el modelo de la síntesis disyuntiva («la no relación como una relación más profunda»: Cfr. Deleuze, 2005, series 7ª $24^{\text {a }}$; Zourabichvili, 2007, 19-20; Deleuze, 1987, 89-98).

Conviene ahora destacar el significativo viraje con respecto al marco teórico descrito que supone el concepto de agenciamiento bosquejado por Deleuze y Guattari en Mil Mesetas; en cuya fragua la influencia de Foucault no es, en absoluto, desdeñable (Cfr. Zourabichvili, 2007, 20). Con miras a extender sus anteriores investigaciones al campo social, Deleuze realiza junto a Guattari una ambiciosa crítica de la lingüística que reivindica la primacía de la pragmática, en tanto que "política de la lengua" de la que depende toda semántica, y tiene el concepto de agenciamiento de enunciación como hilo conductor (Cfr. Deleuze \& Guattari, 1994, "Los postulados de la lingüística"; Zourabichvili, 2007, "Agenciamiento", especialmente 19-20). Entendiendo que la función elemental del lenguaje es la transmisión de consignas, definida la consigna como la relación entre los enunciados y los actos incorporales que se realizan en ellos (ilocutorio: interrogo al preguntar, prometo al dar mi palabra etc) (Cfr. Deleuze \& Guattari, 1994, 83, 84 y siguientes), redefine el atributo lógico como un acto o una transformación instantánea e incorporal que, sin embargo, se atribuye a los cuerpos de una sociedad. El agenciamiento colectivo de enunciación es, entonces, una unidad superior, compuesta de un conjunto de atributos incorporales en tanto que variables (Cfr. Deleuze \& Guattari, 1994, 85, 86, 88); la cual constituye una instancia actual que regula la producción de subjetividades y significados en el campo social, dando lugar a regímenes de signos que permanecen constantes en el tiempo (Cfr. Deleuze, 1994, 84, 88-90); hay aquí cierto aire de semejanza con el concepto foucaultiano de dispositivo: Cfr. Deleuze, 1987, 55, 63). Tales inquisiciones suscitan el concepto, más amplio, de agenciamiento, que está, asimismo, atravesado por dos ejes que lo dividen en cuatro partes: el primero lo 
divide en forma de contenido o agenciamiento maquínico de cuerpos y forma de expresión o agenciamiento colectivo de enunciación; división que traduce la dualidad estoica entre las mezclas de cuerpos y los acontecimientos incorporales, haciéndola aterrizar en la historia y conformando el estrato del saber. El segundo eje se corresponde, en cambio, con los movimientos a que está expuesto el agenciamiento: movimientos de reterritorialización, que lo establecen, y movimientos de desterritorialización, que lo arrastran y desestabilizan, sea para destruirlo o transformarlo.

Fiel a la tradición estoica en que se inspira, la distribución del estrato histórico del saber a lo largo de los dos segmentos que componen el primer eje del agenciamiento ha de presentar una observancia rigurosa del proceso de la síntesis disyuntiva (relegando el ámbito donde rigen los principios lógicos de identidad, no contradicción y tercero excluido a un orden posterior) que da lugar a las series divergentes: entre los enunciados y las visibilidades, el discurso y los estados de cosas, media un abismo insalvable; exigencia tanto de la inmanencia como de la univocidad del ser, principales compromisos que asume la ontología diferencial del autor. Y ello de modo que no se habla de aquello que se ve y no se ve aquello de lo que se habla: todo lenguaje es, como adelantamos, discurso indirecto o transmisión de consignas (Cfr. Deleuze \& Guattari, 1994, 90-93).

Ahora bien, la independencia entre las formas de contenido y expresión lleva aparejada su presuposición recíproca, en una génesis conjunta que remite al diagrama de poder o las relaciones de fuerzas (Cfr. Zourabichvili, 2007, 19). Y ello de modo que:

Si existe primacía del poder sobre el saber, es porque las dos formas heterogéneas del saber se constituyen por integración y entran en una relación indirecta, por encima de su intersticio o su "no relación", en condiciones que sólo corresponden a las fuerzas (Deleuze, 1987, p. 111).

El ámbito del poder o las relaciones de fuerza, cuya naturaleza es virtual y diagramática, antecede ontológicamente a las distribuciones de las formas de contenido y expresión en el estrato histórico del saber, de modo que las genera y determina su posterior organización. Luego, el diagrama de poder condiciona las relaciones estratificadas del saber en las que deviene cognoscible, habida cuenta de su naturaleza volátil y cambiante. Entre el saber y el poder se establece, pues, una compleja relación, de acuerdo con la cual el poder aparece como condición de posibilidad de un saber que, una vez constituido, captura y tiende a fijar las relaciones de poder que lo suscitan. Tal es el cometido de la institución: organizar el poder -cuya naturaleza es molecular- en torno a una instancia molar, es decir, organizar las relaciones de fuerzas en torno a formas estratificadas del saber (a modo de ejemplo: en la institución Prisión, la forma-Prisión distribuye en el espacio y el tiempo los cuerpos de los presos, dando lugar a una determinada visibilidad o evidencia de la delincuencia en una época histórica; y el derecho penal, en cuanto conjunto o familia de enunciados, proporciona fundamento discursivo a tales prácticas). En tanto que institución, todo saber responde en primera instancia a un determinado diagrama de poder que, una vez constituido, tiende a fijar en el tiempo. Así las cosas, «el poder, considerado abstractamente, ni ve ni habla. Es un topo (...) Pero precisamente porque ni habla ni ve, hace ver y hablar». (Deleuze, 1987, 111). 
Dilucidado el marco conceptual, pasamos a considerar la tesis fundamental del trabajo: la Imagen Dogmática da al traste con la lógica de la síntesis disyuntiva que requieren las series heterogéneas. De acuerdo con la presuposición de una relación natural entre el pensar y lo pensado, hace coincidir la forma de contenido y la forma de expresión, en la medida en que abre una visibilidad en el enunciado y un enunciado en la visibilidad, de manera que ambas series resultan unidas en una sola. Esto hace de las series divergentes algo estático, dado de una vez por todas en la distribución del estrato histórico del saber, por cuanto que imposibilita sus movimientos de desterritorialización y reterritorialización relativos, que hacen evolucionar el estrato histórico del saber y, por ende, traducen a nivel molar o institucional las alteraciones que se producen en los devenires de las relaciones de poder establecidas, de naturaleza molecular o intensiva, con los que entran efectivamente en comunicación a través de un "encuentro". ${ }^{2}$ El siguiente fragmento resulta sumamente ilustrativo con respecto a los movimientos de desterritorialización y reterritorialización relativos que ponen en comunicación a las formas de contenido y expresión:

Las formas de expresión y contenido son inseparables de un movimiento de desterritorialización que las arrastra. Expresión y contenido, cada uno de ellos está más o menos desterritorializado, relativamente desterritorializado según tal estado de su forma. A este respecto no se puede plantear una primacía de la expresión sobre el contenido o a la inversa. Puede suceder que las componentes semióticas estén más desterritorializadas que las componentes materiales, pero también puede suceder lo inverso... En resumen, hay grados de desterritorialización que cuantifican las formas respectivas, y según los cuales los contenidos y las expresiones se conjugan, alternan, se precipitan recíprocamente, o, al contrario, se estabilizan al efectuar una reterritorialización (Deleuze \& Guattari, 1994, 92: el subrayado es mío).

En tanto que institución, el saber desarrollado al abrigo de la Imagen Dogmática del pensamiento tiende entonces al estatismo. Y la filosofía, que es aquella forma particular del saber que atañe al pensamiento tal y como es de derecho, permanece atada al sentido común y no alcanza a erigirse en verdadera crítica (esto es, no alcanza a comprender que el saber tiende a fijar las relaciones de poder y, consecuentemente, a plantear la crítica en términos de nuevos valores, contraponiéndolos a los establecidos: Nietzsche constituyó la filosofía como crítica genealógica al introducir en el pensamiento los conceptos de

2 Huelga decir que existe analogía entre la puesta en comunicación de las formas de contenido y de expresión que constituyen el estrato histórico del saber y el diagrama que expresa las relaciones de poder o el cortocircuito de la misma y el concepto deleuzeano de "necedad" en su doble acepción: de un lado, significa la desconexión entre el pensamiento y las relaciones de fuerzas o el poder, esto es, entre el pensamiento y sus condiciones de posibilidad; del otro, la conexión efectiva entre el pensamiento y la fuerza en tanto que objeto límite del pensar, a un tiempo aquello que sólo puede ser pensado y lo impensable mismo, que es condición de posibilidad de todo ejercicio empírico del pensamiento. De acuerdo con la segunda acepción, el concepto de necedad que es inicialmente negativo pasa a adquirir, en un segundo momento, un sentido positivo, constituyendo ahora el punto de partida del pensar. De lo dicho se sigue que, estableciendo una analogía con lo anterior, podríamos hablar en lo que concierte a los procesos de cambio socio-político de una "necedad política" que significaría, de un lado negativo, la desconexión entre el estrato histórico del saber y las relaciones de poder que bloquea el cambio político y, de un lado positivo, la conexión efectiva entre ambos que inaugura la posibilidad del mismo. 
"sentido" y "valor", el primero los cuales remite a la fuerza y el segundo a su elemento diferencial, refiriendo en última instancia la epistemología a la axiología, el saber a la voluntad o las relaciones de poder), sino que se limita a constituir una suerte de refrendo racional de los valores en curso y los estados de cosas existentes, esto es, del statu quo de cada época. Luego, la Imagen Dogmática del pensamiento encierra, tras un aparente potencial crítico fundado en el ejercicio de la razón, un irrefrenable afán justificador a propósito de toda opinión, de toda costumbre socialmente establecida. Calca el derecho que defiende de los hechos del momento, el deber-ser del ser, derivando en una afirmación sin reservas de todo statu quo:

la imagen del pensamiento no es sino la figura bajo la cual se universaliza la doxa elevándola al nivel racional (...) se sigue siendo prisionero de la misma caverna o de las ideas de la época con las que uno sólo se permite la coquetería de "reencontrarlas", bendiciéndolas con el signo de la filosofía (Deleuze, 2006, 208).

\section{Condiciones de posibilidad del cambio socio-político}

Por el contrario, la observancia de la síntesis disyuntiva, a través de cuyos cauces discurren las series divergentes que constituyen la parcela de realidad que nos resulta cognoscible (el saber), es condición de posibilidad de la comunicación efectiva entre el pensamiento y su afuera, el estrato del saber y la realidad intensiva que es el poder, la historia y el devenir, lo molar y lo molecular... Y, por ende, del cambio socio-político. La ruptura con la redistribución unitaria de los cuerpos o visibilidades y las familias de enunciados o decibilidades realizada por la Imagen Dogmática del pensamiento propicia, pues, la comunicación de las series heterogéneas en los movimientos de desterritorialización y reterritorialización relativos que arrastran el agenciamiento. Y ello puesto que tal ruptura supone la existencia de una diferencia de naturaleza y de una mutua irreductibilidad entre las formas de contenido y expresión. Leamos nuevamente a Deleuze:

Podría concebirse una acción causal entre el contenido o la expresión, pero no para las formas respectivas, la forma de contenido y la forma de expresión. A ésta hay que reconocerle una independencia que permitirá precisamente a las expresiones actuar sobre los contenidos (Deleuze \& Guattari, 1994, 93. El subrayado es mío). ${ }^{3}$

3 La independencia de las series heterogéneas presupuesta en el concepto de casi-causa supone: de un lado, contra el reduccionismo materialista, que el efecto incorporal adquiere autonomía y productividad propias al conquistar una causalidad autónoma o casi-causalidad, no quedando subsumido en la causa corporal; del otro lado, contra un enfoque idealista, una ruptura con el mundo representativo del idealismo y el racionalismo, que vislumbraba un enunciado en la visibilidad y una visibilidad en el enunciado, haciendo coincidir las formas de contenido y expresión e imposibilitando, por ende, su desfase relativo (movimientos de reterritorlización y desterritorialización relativos que permitían a la expresión actuar sobre el contenido, anticipándolo). La primera implicación descrita funda la productividad del producto, esto es, del efecto incorporal o el orden de lo simbólico, y ello tanto a nivel del sentido-efecto -forma de expresión- como al del cuerpo-causa -forma de contenido-. Productividad del producto que, de acuerdo con lo dicho, puede ejercerse, en suma, incluso sobre el propio productor. Aquí radica una de las principales rupturas con el marxismo tradicional que Deleuze y Guattari llevan a cabo en $\mathrm{Mil}$ Mesetas: la rígida separación entre la super-estructura y la infraestructura, es decir, entre un ámbito superfi- 
Expresando el atributo no corporal, y atribuyéndolo al mismo tiempo al cuerpo, no se representa, no se refiere, en cierto sentido se interviene, y es un acto de lenguaje... La independencia de la forma de contenido y la forma de expresión no funda ningún paralelismo entre las dos, ni tampoco ninguna representación de la una por la otra, sino, al contrario, una fragmentación de las dos, una manera de insertarse las expresiones en los contenidos, de pasar constantemente de un registro a otro, de actuar los signos sobre las cosas, al mismo tiempo que éstas se extienden y se despliegan a través de los signos. Un agenciamiento de enunciación no habla "de las" cosas, sino que habla desde los mismos estados de cosas o estados de contenidos... En resumen, la independencia funcional de las dos formas sólo es la forma de su presuposición recíproca, del paso incesante de la una a la otra (Deleuze \& Guattari, 1994, 91. La cursiva es mía).

Tal comunicación es anticipada y propiciada en el acontecimiento, entendido por Deleuze como un atributo incorporal que es expresado en los enunciados y, no obstante, se atribuye a los cuerpos; y ello no para representarlos, sino para intervenir en ellos. El proceso de la subjetivación política resulta, asimismo, paradigmático a propósito del frecuente primado de la enunciación a la hora de suscitar los movimientos de desterritorialización relativos que dan lugar al cambio socio-político; espontaneidad del acontecimiento-enunciado que contrasta con la receptividad del cuerpo-visibilidad, reproduciendo a otro nivel la propensión a afectar o a ser-afectado que distingue a las fuerzas (intensidades diferenciales) cuyas relaciones dan lugar al campo diagramático del poder (el campo trascendental reformulado como un plano de inmanencia, que constituye el afuera de que el pensamiento extrae la necesidad que le es propia) (Deleuze, 1987, 106).

En el modelo del pueblo por venir, el primado de la enunciación se presenta explícitamente como una forma de anticipación (...) La subjetivación política aparece entonces no como una forma sin contenido, sino como una forma de expresión a la cual no corresponde -todavía- ninguna forma de contenido (Rambeau, artículo inédito, la traducción es mía)

Da buena cuenta tanto de la anticipación o el primado de la enunciación respecto al contenido como de la relevancia que los procesos de plegamiento o subjetivación política tienen en a propósito del cambio socio-político el elocuente ejemplo que Deleuze introduce en el siguiente fragmento:

Ya era una transformación incorporal la que había extraído de las masas una clase proletaria en tanto que agenciamiento de enunciación, antes de que se dieran las condiciones de aparición de un proletariado como cuerpo. Genialidad de la I ${ }^{\mathrm{a}}$ Internacional marxista, que "inventa" un nuevo tipo de clase: iproletarios de todos los países, uníos! (Deleuze \& Guattari, 1994, 88).

cial y esencialmente improductivo que se corresponde con la ideología y un ámbito profundo y esencialmente productivo a nivel histórico-social, es abolida en favor de un nuevo estatuto productivo en el sentido anterior atribuido también al ámbito del sentido o lo simbólico (Cfr. Deleuze \& Guattari, 1994, 73-78, 87, 94, 144, 284). 


\section{Conclusiones.}

En ¿Qué es la filosofía? Deleuze y Guattari atribuían al arte y la filosofía la tarea titánica de crear un pueblo; reconociendo, no obstante, que tal empresa desborda con creces las virtualidades de ambas disciplinas (Cfr. Deleuze \& Guattari, 1997, 111). Huelga decir que la lógica que rige esta creación o esta llamada a un pueblo que falta es la anticipación de la forma de expresión con respecto a la forma de contenido, primado de la enunciación que, según hemos visto, anticipa la constitución de cuerpos en ciernes en los acontecimientos -actos instantáneos e incorporales que, no obstante, se atribuyen a los cuerpos de una sociedad- y, por ende, en esa subespecie de los mismos que son los procesos de subjetivación política. Las siguientes palabras de Deleuze y Guattari no dejan lugar a dudas al respecto: «la creación de conceptos apela en sí misma a una forma futura, pide una tierra nueva y un pueblo que no existe todavía» (Deleuze \& Guattari, 1997, 110)

Crear un pueblo hace, por ende, alusión a dar lugar a nuevo sustrato para el pensamiento, liberado al fin del yugo del sentido común (cogitatio natural universalis o presuposición de la recta naturaleza del pensamiento y la buena voluntad del pensador) que falsea la aventura emancipadora del pensar en el re-conocimiento del mundo verdadero de la metafísica; haciéndola, a un tiempo, desembocar en una asunción acrítica del statu quo o los valores en curso de cada época.

Tales consideraciones remiten entonces a aquellas otras en que Deleuze afirmaba que la filosofía no se agota en la comprensión estrictamente filosófica -esto es, realizada únicamente a través de conceptos-, sino que precisa, y aun en la misma medida, del sustrato de una comprensión no filosófica -esto es, llevada a cabo a través de afectos y perceptos-. Es en este sentido que el pensador francés dejó escrito que «la comprensión no filosófica no es una comprensión insuficiente o provisional, es una de las dos mitades, una de las dos alas de la comprensión propiamente filosófica» (Deleuze, 1996, p. 222. La cursiva es mía). De un lado, los afectos se corresponden con nuevas maneras de experimentar; del otro, los perceptos hacen lo propio con nuevas maneras de percibir (Cfr. Deleuze, 1996, 232). En ambos casos, el adecuado concurso de las facultades corporales es determinante.

Por otra parte, la pretendida inocencia política de la Imagen Dogmática del pensamiento desaparecerá a poco que advirtamos las concomitancias que existen entre la misma y la forma-Estado, esto es, el hecho de que la Imagen Dogmática y la unificación de las facultades que opera a nivel del Cogito (sujeto-pensamiento elevado al estatus de meta-facultad, cuyos contenidos unitarios le son dados por el trabajo conjunto de las distintas facultades bajo el concierto del sentido común) están inspirados en los órganos de poder del Estado, y ello de modo que «el sentido común, la unidad de todas las facultades como centro del Cogito, es el consenso de Estado llevado al absoluto» (Deleuze \& Guattari, 1994, 381). El pensamiento, como muestra la noología, ha de ser tomado muy en serio (Cfr. Deleuze \& Guattari, 1994, 381).

A la luz de lo dicho anteriormente, esta última constatación pone de manifiesto que se precisan nuevas maneras de percibir y experimentar (nuevos usos de las facultades corporales) como condición de posibilidad de una nueva manera de pensar: «la filosofía tiene que devenir no filosofía, para que la no filosofía devenga la tierra y el pueblo de la filosofía» (Deleuze, Guattari, 1997: 111). Los afectos y los perceptos constituyen, por ende, un sustrato 
no-filosófico de la filosofía o un afuera del pensamiento. Y es preciso, en suma, sustraer el cuerpo y sus facultades al gobierno de la Imagen Dogmática, suscitando nuevas maneras de percibir y experimentar que den lugar, a su vez, a un nuevo modo de pensar. Tal es, en última instancia, el sentido de la llamada a un pueblo que falta; en la que, como hemos visto, el acontecimiento y la subjetivación política juegan un papel sumamente determinante, hasta el punto de devenir en condición de posibilidad tanto del pensamiento como de la resistencia.

\section{Bibliografía}

\section{Bibliografía Principal:}

Deleuze, Gilles, (1996): Conversaciones. 1972-1990 (2a ed.). Valencia: Pre-Textos.

Deleuze, Gilles, (2006): Diferencia y repetición, Trad. María Silvia Delpy y Hugo Beccacece, Buenos Aires: Amorrortu (edición original 1968).

Deleuze, Gilles, Alcalá Rodríguez, Francisco Javier. "De Luces Y Sombras. La Dualidad «Naturaleza/Civilización» En La Pintura de Caspar Friedrich Como Antecedente de El Corazón de Las Tinieblas, de Joseph Conrad," 33-47, 2015.

Deleuze, Gilles (2005): Lógica del sentido, Trad. Miguel Morey y Víctor Molina, Barcelona: Paidós (edición original 1969).

Deleuze, Gilles, Guattari, Felix (1997): ¿Qué es la filosofía?, Trad. Thomas Kauf, Barcelona: Anagrama (edición original 1991).

Deleuze, Gilles, Guattari, Felix, (1994): Mil mesetas: capitalismo y esquizofrenia, Trad. José Vázquez Pérez y Umbelina Larraceleta, Valencia: Pre-Textos (edición original 1980).

\section{Bibliografía Secundaria:}

Bréhier, Émile (1928): La Théorie des incoroporels dans l'ancien stoicïsme, Paris: Librairie Philosophique J. Vrin.

Nietzsche, Friedrich (1980): Crepúsculo de los ídolos, Madrid: Alianza Editorial.

Rambeau, Fréderic (2014): Deleuze, Guattari et les apories de la subjectivation politique, Artículo inédito (la traducción es mía).

Zourabichvili, François (2004): Deleuze. Una filosofía del acontecimiento, Buenos Aires : Amorrortu.

Zourabichvili, François (2007): El vocabulario de Deleuze, Buenos Aires: Atuel. 